\title{
Aggressive Fluid Resuscitation in Severe Pediatric Hyperglycemic Hyperosmolar Syndrome: A Case Report
}

\author{
Srinivas Murthy ${ }^{1}$ and Rana Sharara-Chami ${ }^{2}$ \\ ${ }^{1}$ Department of Pediatrics, Children's Hospital Boston, 300 Longwood Avenue, Boston, MA 02115, USA \\ ${ }^{2}$ Department of Anesthesia, Children's Hospital Boston, 300 Longwood Avenue, Boston, MA 02115, USA
}

Correspondence should be addressed to Srinivas Murthy, sgmurthy@gmail.com

Received 9 November 2009; Accepted 26 January 2010

Academic Editor: Durval Damiani

Copyright (c) 2010 S. Murthy and R. Sharara-Chami. This is an open access article distributed under the Creative Commons Attribution License, which permits unrestricted use, distribution, and reproduction in any medium, provided the original work is properly cited.

\begin{abstract}
Objective. This report describes a severe case of hyperglycemic hyperosmolar syndrome complicated by rhabdomyolysis, acute kidney injury, hyperthermia, and hypovolemic shock, with management centred upon fluid administration. Design. Case report. Setting. Pediatric intensive care unit in university teaching hospital. Patients. 12 years old adolescent female presenting with hyperglycemic hyperosmolar syndrome with a new diagnosis of type 2 diabetes mellitus. Intervention. Aggressive fluid resuscitation and insulin. Main results. The patient had a good outcome, discharged home on hospital day 6. Conclusions. Hyperglycemic hyperosmolar syndrome is associated with a number of complications. Management strategies are undefined, given the rarity of its presentation, and further studies are warranted.
\end{abstract}

\section{Introduction}

Recent trends indicate a rising percentage of type two diabetes in the under eighteen population, with a prevalence of 0.22 cases per 1000 youth [1] amongst the American population. Hyperglycemic hyperosmolar syndrome (HHS), a rare pediatric complication of diabetes mellitus (DM), is characterized by the triad of hyperglycemia (typically $>600 \mathrm{mg} / \mathrm{dL}$ ), hyperosmolality (serum osmolality $>330 \mathrm{mOsm} / \mathrm{L}$ ), and a mild metabolic acidosis $(\mathrm{pH}>7.2)$. It portends a very poor prognosis, with mortality rates of between $10-50 \%$ in adults [2]. In children, similar mortality rates have been observed [3], although most of the literature is limited to case series and reports. In adults, documented major complications include thrombosis, rhabdomyolysis, renal failure, and irreversible cardiac arrhythmias [4]. A formal distinction between HHS and diabetic ketoacidosis (DKA) exists, with DKA typically presenting with lower levels of hyperglycemia, ketosis, and more significant metabolic acidosis, although these two illnesses are more likely on the spectrum of decompensations related to diabetes [2]. HHS is classically associated with type $2 \mathrm{DM}$, although it has also been increasingly reported in type $1 \mathrm{DM}[5,6]$ as well. DKA has a much better prognosis, with pediatric mortality rates below $1 \%$ [7], and cerebral edema being the major cause of death.

We present a case of an adolescent girl with newly diagnosed HHS and review some of the current literature on the presentation and management of pediatric HHS.

\section{Case Presentation}

The patient was a previously healthy 12 year-old African American female who presented to the emergency room with a chief complaint of altered mental status. She had been having upper-respiratory symptoms for the preceding 4-5 days, with diarrhea and vomiting for three days. She was thirsty, supplementing her diet with milkshakes and smoothies. The night prior to presentation, she awoke somewhat disoriented, which the parents assumed was a bad dream. On the day of presentation, the patient became progressively weak and sleepy, before presenting to the emergency room with lethargy.

Review of systems was notable for mild polydipsia, some nocturia over the past few nights, no fevers, and no myalgia. 
There is a paternal family history of type $1 \mathrm{DM}$, and no history of gestational diabetes. There is a significant family history for obesity.

Upon presentation, the patient had a Glasgow Coma Scale of 7. Vital signs were as follows: heart rate $159 \mathrm{bpm}$, respiratory rate of 20 breaths per minute, and temperature of $104.4^{\circ} \mathrm{F}\left(40.2^{\circ} \mathrm{C}\right)$. Blood pressure was $79 / 37 \mathrm{~mm} \mathrm{Hg}$. Weight was $65 \mathrm{~kg}$, and BMI was $26 \mathrm{~kg} / \mathrm{m}^{2}$ (80th percentile for age). An initial rapid glucose test read $>750 \mathrm{mg} / \mathrm{dL}$. Pupils were equal and responsive to light. Mucus membranes were dry. The neck was supple with no jugular venous distension and notable for acanthosis nigricans. There were decreased breath sounds on the right base, with some nasal flaring, but no significant respiratory distress. Cardiac examination was consistent with sinus tachycardia and no murmurs. Pedal pulses were minimally palpable. Abdomen was obese, soft and non-distended, with no hepatosplenomegaly. Striae were present on the flanks.

Initial laboratory evaluation revealed: arterial blood gas pH 7.12; $\mathrm{PCO}_{2} 50 \mathrm{mmHg} ; \mathrm{HCO}_{3} 15 \mathrm{mM} ; \mathrm{PaO}_{2} 99 \mathrm{mmHg}$. Complete blood count had a white blood count of $14.6 / \mu \mathrm{L}$, a hematocrit of $37 \%$ and a platelet count of $507,000 / \mu \mathrm{L}$. Electrolytes were the following: sodium $(\mathrm{Na}): 137 \mathrm{mM}$ (corrected $\mathrm{Na}$ using the formula: Corrected $\mathrm{Na}=$ Measured $\mathrm{Na}+1.6$ (glucose $(\mathrm{mg} / \mathrm{dL})-100$ ) was $185 \mathrm{mM}$ ); potassium: $5.2 \mathrm{mM}$; chloride: $102 \mathrm{mM}$, phosphorus: $4.5 \mathrm{mg} / \mathrm{dL}$. Serum glucose: 2636 mg/dL, Osmolality: 466 mOsm/L; BUN: 77 mg/dL; Creatinine: $4.08 \mathrm{mg} / \mathrm{dL}$; troponin I $0.268 \mathrm{ng} / \mathrm{ml}(N<0.1)$; lactic acid: $7.6 \mathrm{mM}$, amylase $112 \mathrm{U} / \mathrm{L}$; hemoglobin A1C 11.5\%; liver enzymes and coagulation profile were normal; islet cell antibody: negative; glutamic acid decarboxylase antibody: negative; urinalysis with $3+$ glucose and trace ketones.

A chest $X$-ray was unremarkable. Because of the high fever and the altered mental status, a head CT and a lumbar puncture were performed. The CT was not consistent with cerebral edema. Lumbar puncture had an opening pressure of $30 \mathrm{~cm}$ of $\mathrm{H}_{2} \mathrm{O}$ and a closing pressure of $27 \mathrm{~cm}$ of $\mathrm{H}_{2} \mathrm{O}$. CSF studies revealed: $0 \mathrm{RBC}, 1 \mathrm{WBC}$, protein $51 \mathrm{mg} / \mathrm{dL}$, glucose $869 \mathrm{mg} / \mathrm{dL}$, and a negative gram stain.

The patient received $3 \mathrm{~L}$ of normal saline intravenously over a span of three hours in the emergency room, with some improvement in mental status and blood pressure. She was started on a regular insulin drip at $0.1 \mathrm{U} / \mathrm{kg} / \mathrm{hour}$ and was transferred to the pediatric intensive care unit.

In the PICU, the patient persisted in hypovolemic shock and required aggressive fluid resuscitation with approximately $100 \mathrm{~mL} / \mathrm{kg}$ of isotonic fluid boluses over the first 12 hours, as well as dopamine with maximal dosing of $5 \mathrm{mcg} / \mathrm{kg} / \mathrm{min}$ for hemodynamic support. She was started on intravenous hydration with $154 \mathrm{mEq} / \mathrm{L}$ of sodium acetate with added potassium chloride and potassium phosphate. Vancomycin and ceftriaxone were initiated. She was placed on BiPAP for ventilatory support due to persistent desaturations. She was started on enoxaparin for DVT prophylaxis. Glucose decreased at a rate of approximately $130 \mathrm{mg} / \mathrm{dL} /$ hour, with an increasing serum sodium (Figure 1). Mental status continued to improve and 12 hours post-presentation, she responded to commands and was oriented to place and person. She was weaned off

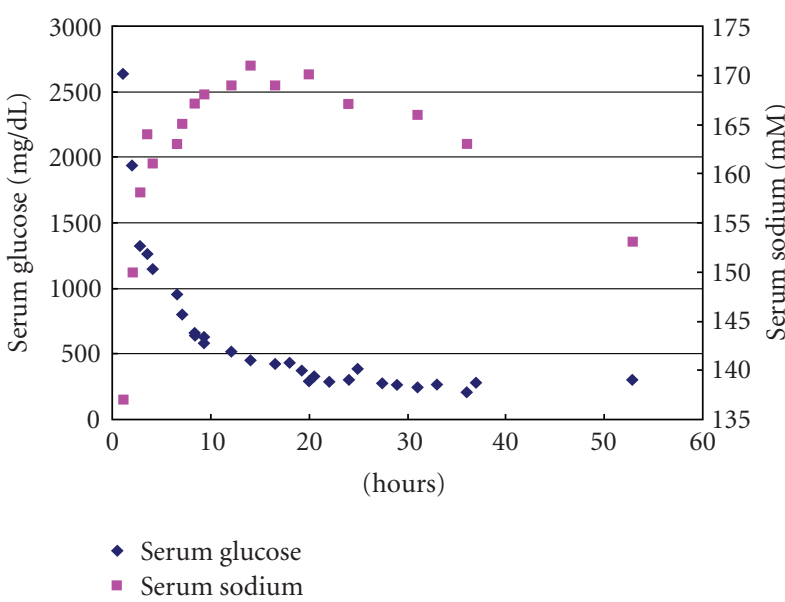

FIgURE 1: Serum glucose and sodium during hospitalization.

hemodynamic and ventilatory support, but continued to require fluid resuscitation with only slow improvement in serum lactic acid levels, receiving a total of $20 \mathrm{~L}$ of isotonic fluid in the first 36 hours of admission. As mental status recovered, she transitioned off the insulin infusion to a subcutaneous regimen. All laboratory values and vital signs continued to improve, including serum troponin, BUN, and creatinine and urine output continued to be brisk. She reported some myalgia, and a CK was $4051 \mathrm{IU} / \mathrm{L}$ at 36 hours post-presentation. She was transitioned to a full diet within 48 hours of presentation. Antibiotics were discontinued after blood, urine, and CSF cultures were negative at 48 hours. She maintained an intact neurological examination throughout her hospitalization. She was transferred to the regular floor after a 60 hours PICU admission, and was discharged home on a glargine-containing insulin regimen after a total six-day hospital course.

\section{Discussion}

This patient presented with HHS as a complication of newly diagnosed type 2 diabetes mellitus. The diagnosis of type $2 \mathrm{DM}$ was made based on the lack of pancreatic auto-antibodies, historical and physical characteristics, and a positive family history for type $2 \mathrm{DM}$. Her presentation is very similar to previously reported cases of extreme hyperglycemia in pediatric HHS $[8,9]$, although her initial serum glucose was one of the highest reported in the pediatric population. The absence of earlier symptoms is emblematic of the classical presentation of HHS, where the absence of ketosis and acidosis delays the subjective illness [10], in contrast with the rapid presentation of its counterpart, DKA [11]. Her initial presentation was also notable for a metabolic acidosis, likely explained by an elevated lactic acid level secondary to profound dehydration and decreased end-organ perfusion. Her significant hyperthermia at presentation is a published complication of HHS [9], whose etiology is unknown, but potentially related to rhabdomyolysis. Contrary to previous reports [12], her temperature developed prior to administration of 
insulin, negating the insulin preservative $\mathrm{m}$-cresol as the etiology of her hyperthermia. Undiagnosed inborn errors of metabolism, severe physiologic stress, or undiagnosed infection are unlikely reasons for her initial hyperthermia.

Hyperglycemic hyperosmolar state occurs when progressive insulin resistance worsens in the setting of some physiologic stress, driving up serum glucose while still retaining an effect on the suppression of lipolysis. Given the hormonal sensitivity of the adipocyte, ketosis is suppressed with the residual activity of insulin. An upregulation in glucagon release secondary to the decreased activity of insulin contributes to hepatic gluconeogenesis, further augmenting the hyperglycemic state $[13,14]$. The worsening hyperglycemia hastens osmotic diuresis, leading to hypernatremic dehydration, as excess water is lost with respect to salt. Once renal glucose clearance capacity is overwhelmed and GFR becomes compromised, a hyperosmolar state develops, leading to the clinical signs and symptoms, including the altered mental status and profound dehydration.

Given the severe dehydration, often estimated to be up to $20 \%$ of body volume, and residual insulin activity in HHS, management strategies centre upon fluid administration. Evidence based guidelines in children are lacking, so data are extrapolated from adult guidelines, children with DKA, and the few case series of pediatric HHS. What makes this case notable was the degree of aggressive fluid resuscitation, with the patient receiving nearly $20 \mathrm{~L}$ of intravenous fluids within the first 36 hours of hospitalization, guided by hypotension and lactic acidemia. Guidelines for adults recommend rapid correction of serum osmolality towards a goal of $<320 \mathrm{mOsm} / \mathrm{L}$ [4]. In children, some caution is theoretically warranted, and the fluid administration rate should be tempered by this risk, with a goal reduction in serum glucose of $100-150 \mathrm{mg} / \mathrm{dL} /$ hour. In some pediatric reports, however, poor outcomes have been associated with minimizing fluid repletion [15], arguing that HHS in children is similar to HHS in adults, where aggressive fluid resuscitation is the rule $[2,4]$. A previous case of extreme hyperosmolality has been reported with no significant rise in measured intracranial pressure with a relatively rapid correction of serum osmolality [16]. Isotonic solution should be considered to temper the risk of excessive changes in osmolality until definitive data are obtained, and any hypovolemic shock should be corrected aggressively. This is in strict contrast to management guidelines for pediatric DKA, where the degree of dehydration is much less, and fluid repletion should be more carefully administered, given the documented risk of cerebral edema [17-19].

Pediatric HHS patients are classically quite sensitive to exogenous insulin, so standardized infusion rates of $0.1 \mathrm{U} / \mathrm{kg} /$ hour are recommended. Dextrose is added to intravenous fluids appropriately to avoid overly precipitous drops in glucose levels. Upon adequate control of serum glucose, insulin administration should be switched to an appropriate subcutaneous regimen. Potassium, phosphate, and calcium need to be monitored closely, given the risks of abnormalities through both the pathogenesis and treatment of HHS. Muscle enzymes should be empirically checked in every patient with HHS, given the risk of rhabdomyolysis and its effects on management, considering data on alkalinization and outcomes in DKA [19]. Also, venous sinus thrombosis is a potential complication of severe hypernatremia and hyperosmolality [20], hence the requirement for hydration and careful control of serum sodium as well as for frequent neurological examinations. The risk of other venous thrombotic phenomena, such as pulmonary emboli or deep vein thrombosis [21], are significant, and these should be closely monitored.

We report an extreme case of pediatric HHS with a good outcome. Our patient had many of the known complications of HHS, including hypovolemic shock, rhabdomyolysis, acute kidney injury, and hyperthermia, as well as the one of the highest published serum glucose levels in the pediatric literature. She responded well to very aggressive fluid resuscitation and gentle insulin administration. Prospective trials in this burgeoning population need to be performed so as to better codify management strategies, especially pertaining to the intensity of fluid repletion. Although an uncommon presentation in the pediatric population, clinicians should be aware of the manifestations of HHS and the differences in management from its counterpart, DKA.

\section{References}

[1] A. D. Liese, R. B. D’Agostino Jr., R. F. Hamman, et al., "The burden of diabetes mellitus among US youth: prevalence estimates from the SEARCH for Diabetes in Youth Study," Pediatrics, vol. 118, no. 4, pp. 1510-1518, 2006.

[2] M. F. Magee and B. A. Bhatt, "Management of decompensated diabetes: diabetic ketoacidosis and hyperglycemic hyperosmolar syndrome," Critical Care Clinics, vol. 17, no. 1, pp. 75-106, 2001.

[3] J. B. Cochran, S. Walters, and J. D. Losek, "Pediatric hyperglycemic hyperosmolar syndrome: diagnostic difficulties and high mortality rate," American Journal of Emergency Medicine, vol. 24, no. 3, pp. 297-301, 2006.

[4] R. Matz, "Management of the hyperosmolar hyperglycemic syndrome," American Family Physician, vol. 60, no. 5, pp. 1468-1476, 1999.

[5] A. Basso, L. Dalla Paola, G. Erle, D. Nacamulli, and D. Armanini, "Hyperosmolar nonketotic coma at the onset of type I diabetes in a child," Journal of Endocrinological Investigation, vol. 20, no. 4, pp. 237-239, 1997.

[6] M. F. Canarie, C. W. Bogue, K. J. Banasiak, S. A. Weinzimer, and W. V. Tamborlane, "Decompensated hyperglycemic hyperosmolarity without significant ketoacidosis in the adolescent and young adult population," Journal of Pediatric Endocrinology and Metabolism, vol. 20, no. 10, pp. 1115-1124, 2007.

[7] J. P. Orlowski, C. L. Cramer, and M. R. Fiallos, "Diabetic ketoacidosis in the pediatric ICU," Pediatric Clinics of North America, vol. 55, no. 3, pp. 577-587, 2008.

[8] S. H. Fourtner, S. A. Weinzimer, and L. E. Levitt Katz, "Hyperglycemic hyperosmolar non-ketotic syndrome in children with type 2 diabetes," Pediatric Diabetes, vol. 6, no. 3, pp. 129-135, 2005.

[9] A. S. Hollander, R. C. Olney, P. R. Blackett, and B. A. Marshall, "Fatal malignant hyperthermia-like syndrome with rhabdomyolysis complicating the presentation of diabetes mellitus in adolescent males," Pediatrics, vol. 111, no. 6, pp. 1447-1452, 2003. 
[10] J. L. Chiasson, N. Aris-Jilwan, R. Belanger, et al., "Diagnosis and treatment of diabetic ketoacidosis and the hyperglycemic hyperosmolar state," Canadian Medical Association Journal, vol. 168, no. 7, pp. 859-866, 2003.

[11] D. W. Cooke and L. Plotnick, "Management of diabetic ketoacidosis in children and adolescents," Pediatrics in Review, vol. 29, no. 12, pp. 431-436, 2008.

[12] F. Wappler, N. Roewer, A. Kochling, H. Braune, T. Reissinger, and J. Schulte Am Esch, "Fulminant malignant hyperthermia associated with ketoacidotic diabetic coma," Intensive Care Medicine, vol. 22, no. 8, pp. 809-812, 1996.

[13] R. H. Unger and L. Orci, "Glucagon and the A cell: physiology and pathophysiology (first of two parts)," New England Journal of Medicine, vol. 304, no. 25, pp. 1518-1524, 1981.

[14] R. H. Unger and L. Orci, "Glucagon and the A cell: physiology and pathophysiology (second of two parts)," New England Journal of Medicine, vol. 304, no. 26, pp. 1575-1580, 1981.

[15] R. M. Carchman, M. Dechert-Zeger, A. S. Calikoglu, and B. D. Harris, "A new challenge in pediatric obesity: pediatric hyperglycemic hyperosmolar syndrome," Pediatric Critical Care Medicine, vol. 6, no. 1, pp. 20-24, 2005.

[16] D. D. Vernon and D. C. Postellon, "Nonketotic hyperosmolal diabetic coma in a child: management with low-dose insulin infusion and intracranial pressure monitoring," Pediatrics, vol. 77, no. 5, pp. 770-772, 1986.

[17] I. Fiordalisi, W. E. Novotny, D. Holbert, L. Finberg, and G. D. Harris, "An 18-yr prospective study of pediatric diabetic ketoacidosis: an approach to minimizing the risk of brain herniation during treatment," Pediatric Diabetes, vol. 8, no. 3, pp. 142-149, 2007.

[18] J. A. Edge, R. W. Jakes, Y. Roy, et al., "The UK case-control study of cerebral oedema complicating diabetic ketoacidosis in children," Diabetologia, vol. 49, no. 9, pp. 2002-2009, 2006.

[19] N. Glaser, P. Barnett, I. Mccaslin, et al., "Risk factors for cerebral edema in children with diabetic ketoacidosis. The Pediatric Emergency Medicine Collaborative Research Committee of the American Academy of Pediatrics," New England Journal of Medicine, vol. 344, no. 4, pp. 264-269, 2001.

[20] M. J. Sasiadek, D. Sosnowska-Pacuszko, M. Zielinska, and T. Turek, "Cerebral venous thrombosis as a first presentation of diabetes," Pediatric Neurology, vol. 35, no. 2, pp. 135-138, 2006.

[21] C. R. Keenan, S. Murin, and R. H. White, "High risk for venous thromboembolism in diabetics with hyperosmolar state: comparison with other acute medical illnesses," Journal of Thrombosis and Haemostasis, vol. 5, no. 6, pp. 1185-1190, 2007. 